\title{
Immunosenescence and Respiratory Infections Among Nursing Home Residents
}

\author{
Mark Loeb
}

\section{Contents}

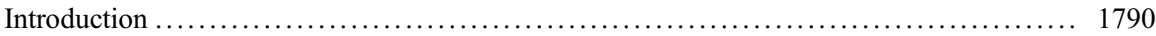

Immunosenescence in the Nursing Home Elderly ............................ 1792

Immune Biomarkers Predictive of Respiratory Viral Infection in Elderly Nursing Home

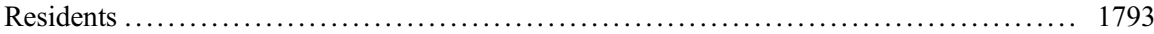

Conclusions and Future Directions ........................................ 1794

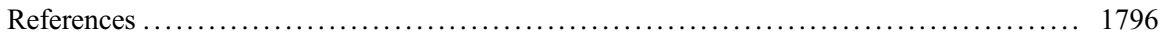

\begin{abstract}
Residents of nursing homes are at high risk of infectious complications due to respiratory infection. The nursing home setting places residents at high risk given the frequent contact among residents and by staff along with the possibility for continuous introduction of respiratory viruses from the community. Nursing home residents are among the most frail members of society. They have multiple comorbidities that can increase their risk of infection. Immunosenescence plays an important role in not only rendering these seniors susceptible in infection, particularly viral respiratory infection, but also interferes with protection. That is, the ability to mount a robust immune response to influenza and pneumococcal vaccine increase the risk. There have been a number of $\mathrm{T}$ cell deficits described in this population. CD4+ T cells, in particular T-regs and CMV-reactive CD4+ $\mathrm{T}$ cells, have been shown to be predictive of respiratory viral infection in this population. Although evidence exists that $\mathrm{T}$ cell subsets may correlate better with
\end{abstract}

M. Loeb $(\bowtie)$

Department of Pathology and Molecular Medicine, McMaster University, Hamilton, ON, Canada

Department of Clinical Epidemiology and Biostatistics, McMaster University, Hamilton, ON, Canada

Institute for Infectious Diseases Research, McMaster University, Hamilton, ON, Canada e-mail: loebm@mcmaster.ca 
response to vaccine and protection, antibody responses to influenza vaccine remains an important correlate in this population. Large-scale epidemiologic studies are needed to establish better correlation between biomarkers for protection and respiratory and other pathogens that circulate in nursing homes.

\section{Keywords}

Nursing homes $\cdot$ Respiratory infection · Influenza $\cdot$ Innate immunity $\cdot$ Clinical studies

\section{Introduction}

It is important to consider why the nursing home environment is so conducive to respiratory infection. A prime factor is that nursing homes are a relatively closed environment. That is, residents live in close quarters and have frequent contact (Strausbaugh et al. 2003). Typically, residents have meals at a common dining area and have activities together. There is a high degree of cognitive impairment among residents of nursing homes, so wandering is common. As a consequence, opportunities for exposure are common. Transmission of respiratory viruses is generally by direct skin-to-skin contact, or by direct contact with mucosal surfaces, or contact with respiratory secretions. Coughing and sneezing can effectively transmit respiratory viruses. Indirect contact through contamination of the environment also plays a role, albeit more limited. The role of staffing is also an important consideration. Generally, staff are assigned to certain units in a nursing homes, but there may be circulation between various floors by some healthcare workers. There are also frequent visitors to nursing homes, all of which serve as entry point for respiratory viruses. Although staff in most facilities are either encouraged or required to be vaccinated against influenza, this is not the case for visitors.

Viral respiratory infections play an important burden to the well-being of nursing home residents. When the types of outbreaks in nursing homes are systematically reviewed, influenza accounts for the largest number of outbreaks by a single pathogen (Utsumi et al. 2010). Respiratory viruses circulate throughout the year in nursing homes. Although unusual, even influenza has been reported during the summer in these facilities. Outbreaks of respiratory infection in nursing homes tend to be explosive, with rapid ascension of cases with subsequent decline (Masse et al. 2016). Generally, the epidemiology of the viruses reflects circulation in the community.

The degree to which respiratory viruses cause primary viral pneumonia among residents of nursing homes is not well understood. Respiratory viruses such as influenza, adenovirus, respiratory syncytial virus, human meta-pneumovirus, and parainfluenza virus are frequently detected as the causative agents of outbreaks in the nursing home settings (Masse et al. 2016; Meijer et al. 2013; Centers for Disease Control and Prevention (CDC) 2013; Todd Faulks et al. 2000). It is assumed that the majority of cases of pneumonia in this setting are due to bacterial superinfection. 
However, it is unclear to what extent serious illness is due to superinfection with bacteria such as pneumococcus since there are no studies that have systematically measured viral infection and subsequent comphrehensive testing for pneumococcus. What is known is that the incidence of invasive disease with pneumococcus among residents of nursing homes is about four times that of age- and sex-matched controls in the community (Kupronis and Richards 2003). While bacterial superinfection is likely for a certain proportion of cases, the epidemiology of primary viral pneumonia in this population remains uncertain. Although pneumonia is treated empirically in this setting with antibiotics that provide coverage for pneumocococcus, there are few options available for the treatment of respiratory viral infection. In fact, at present, only neuraminidase inhibitors, such as oseltamivir, are available for the treatment of influenza infection (McKimm-Breschkin 2005; Gorišek Miksić et al. 2015; Monto et al. 2004).

Relatively few prospective studies exist of the epidemiology of respiratory outbreaks in nursing homes. In one Canadian study, a total of 16 outbreaks were identified during prospective surveillance over 3 years (Loeb et al. 2000). Outbreak days accounted for $9 \%$ of resident-days during the surveillance period. The attack rates ranged from $2 \%$ to $25 \%$ in the nursing homes. Cough, fever, and coryza were the most common symptoms. The clinical findings were not specific for a particular virus and could not be used to distinguish between various viruses. Pathogens identified included influenza A and B (5 outbreaks of influenza A and 2 of influenza B), parainfluenza virus (13 outbreaks), and respiratory syncytial virus (2 outbreaks). In a surveillance study where data was derived from a clinical trial of vitamin $\mathrm{E}$ supplementation in residents of nursing homes, 204 viral infections were documented in $157(25 \%)$ of 617 nursing home residents where human metapneumovirus and coronavirus being the most common etiologies (Meydani et al. 2004).

Influenza vaccination is often said to be the cornerstone for prevention of influenza in this population. However, despite the fact that residents of nursing homes in Canada and the United States tend to be highly vaccinated (with rates typically $>90 \%$ ), outbreaks of influenza continue to be reported (Mitchell et al. 2006; Bowles et al. 2002). Reports of the efficacy of the vaccine in this population are variable.

In one systematic review of observational studies, including 11,262 institutionalized older adults, a significant reduction in pneumonia was observed (vaccine efficacy of 37\%). However, the effect of bias in observational studies of influenza vaccine is well documented (Simonsen et al. 2007; Jackson et al. 2006a, b, 2013; Eurich et al. 2008). A Cochrane systematic review of influenza vaccination that included influenza in long-term care facilities reported similar findings (Rivetti et al. 2006). In homes for elderly individuals (with good vaccine match and high viral circulation), the effectiveness of vaccines against ILI was $23 \%(6 \%$ to $36 \%)$ and nonsignificant against influenza (RR 1.04: $95 \%$ CI 0.43 to 2.51 ). Well-matched vaccines prevented pneumonia (vaccine efficacy $46 \% ; 30 \%$ to $58 \%$ ), hospital admission (vaccine efficacy $45 \% ; 16 \%$ to $64 \%$ ), and deaths from influenza or pneumonia (vaccine efficacy $42 \%, 17 \%$ to $59 \%$ ). 


\section{Immunosenescence in the Nursing Home Elderly}

Although it is generally accepted that elderly residents of nursing homes are a group at high risk for respiratory viral infections and this risk is based on immunosenescence to a considerable degree, there are few studies that have examined immunosenescence in these individuals. Immune phenotypes in the nursing home elderly have not been well described. Although all protective immune mechanisms are influenced by advanced age, alterations in T cells are affected the most (Fulop et al. 2005). There are fewer naïve $\mathrm{T}$ cells because of thymic involution and there is an increase in terminally differentiated memory T cells (Saule et al. 2006).

In a study conducted in three nursing homes, 116 residents, with a median age of 86 years, were evaluated (Fahey et al. 2000). All residents had multiple comorbidity, cognitive impairment, and functional impairment and therefore considered typical of long-term stay frail nursing home residents. The reference young population for immunologic measurements included 21 healthy Caucasian females with median age 38, ranging from 22 to 49, who were tested contemporaneously with the elderly populations. Thirty-three immune parameters were measured. The numbers of total T cells (CD3+), CD4+, and CD8+ subsets were not significantly different from younger reference populations. The B cell (CD19+) percentage was reduced however. CD45RO+ (memory) and CD45RA+ (naive) cells were evaluated and a shift toward memory forms of CD3 T cells was seen with the increased percentage of cells expressing CD45RO. A corresponding reduction in the percentage of CD45RA+ naive $\mathrm{T}$ cells was seen in the CD4 (significant) and CD8 (suggestive) subsets. Functional changes in immune cells were measured by proliferative response following stimulation with phytohemagglutinin.

Proliferative response to phytohemagglutinin in peripheral blood mononuclear cells was decreased in the frail elderly population with significant change to higher dose and suggestive significance of change to lower phytohemagglutinin dose. Significant reduction in CD28 expression (a costimulatory molecule for proliferative stimulation) was seen on the CD3 + T cell. This change was most marked in the CD8+ $\mathrm{T}$ cell subset (67-37\%). The percentage and number of CD3 and CD4 $\mathrm{T}$ cells showed a progressive fall with increasing age. However, there was no notable change for $\mathrm{CD} 8, \mathrm{NK}$, or $\mathrm{B}$ cells nor for proliferative responses to phytohemagglutinin.

A classification tree analysis revealed that increased plasma activation markers (neopterin and sTNF-R) and increased CD28 expression on CD8 T cells and proliferative response separated the aged and control populations. Statistical procedures utilizing principal components analyses, partial correlations, and exploratory factor analyses all indicated that immunologic parameters in frail elderly are grouped in three major clusters of immunologic results. These involved (a) increased plasma levels of neopterin and sTNF receptor indicating elevated IFN $\gamma$ and TNF cytokine activity; (b) increased proportion of mature (CD45RO) versus naïve (CD45RA) T cells; and (c) a diverse group of related changes including impaired proliferative response, reduced T cells, CD28 and CD25 expression, B cell percentage, and lower CD4:CD8 ratios and increased HLA-DR expression. 
The overall observations from this study are compatible with immunosenescent phenotypes seen in community dwelling elderly (Cossarizza et al. 1996; Collerton et al. 2012; Wack et al. 1998). Another study, conducted in Canada, sought to determine the impact of frailty and malnutrition on elderly residents of nursing homes (Johnstone et al. 2014a). Furthermore, the changes to a separate class of T cell, the regulatory CD4+ T cell (T-reg) and to the innate immune natural killer (NK) cell subsets were described in the nursing home elderly.

In the Canadian study, nursing home residents from Ontario were enrolled. Nursing home residents $\geq 65$ years were eligible and those with immunosuppression illnesses or medications were excluded. A sample of healthy adults between the ages of 18 and 65 years were enrolled as comparators. Immune phenotypes, as well as frailty (measured using a clinical frailty scale), and nutritional status was measured. In total, 262 nursing home elderly were enrolled with a median age of 87 years. The vast majority had severe frailty. The majority had at least one co-morbidity, and over half had a diagnosis of dementia. Sixty percent of the participants scored 5 or 6 on the Clinical Frailty Scale, categorizing them as either mildly frail or moderately frail (Rockwood et al. 2007). There were significantly fewer naïve CD8+ T cells, significantly more terminally differentiated CD $8+\mathrm{T}$ cells, and significantly more $\mathrm{CD} 8+$ senescent $\mathrm{T}$ cells in the nursing home population. There were significantly more CD4+ T cells due to more central memory and effector $\mathrm{T}$ cells in the nursing home residents. Having more senescent NK cells was associated with increased frailty and worse nutrition.

While the findings of studies that compare frail elderly populations to healthy seniors or to younger populations are of clear importance, one of the limitations is the clinical relevance of the biomarkers. A cohort study which attempted to address this is described below.

\section{Immune Biomarkers Predictive of Respiratory Viral Infection in Elderly Nursing Home Residents}

Despite the burden of influenza and other respiratory viruses in residents of nursing homes, there are few studies that have systematically examined whether immune biomarkers can predict respiratory viral infection. This is likely because of the number of challenges of doing such clinical studies in nursing home residents. First, informed consent is required, and this often is obtained from substitute decision makers, namely family members, because of the high prevalence of cognitive impairment in this population. Secondly, the timing of obtaining and processing PBMCs is difficult. Thirdly sufficient resources are required both for research nurses and laboratory technologists and reagents. To date, there is only one study which overcame these challenges to generate immune biomarker data and is described below.

This study was conducted in 32 Canadian nursing homes over a 3-year period (Johnstone et al. 2014b). Peripheral blood mononuclear cells (PBMCs) were obtained after vaccination against influenza in a cohort of 1,072 residents. These 
specimens were then tested by flow cytometry for CD4+ T cell and CD8 $+\mathrm{T}$ cell subsets (CCR7 + CD45RA+, CCR7-CD45RA+ and CD28-CD57+) as well as T-regs and CMV-reactive CD4+ T and CD8+ T cells. The concept was to use these baseline markers as predictor variables in statistical models to determine which markers predicted respiratory viral infection. Prospective surveillance was then conducted and the incidence of viral respiratory viruses was determined. A time to event analysis was conducted to determine the relationship between immune phenotypes and time to symptomatic viral infection. Of a total of 1165 residents enrolled, 1087 (93\%) had PBMCs obtained. The median age of this population was 86 years and about half scored with 7 or 8 on the Clinical Frailty Scale, which represents severe frailty (Rockwood et al. 2007). There were 87 residents who had laboratory confirmed viral respiratory illness, with corona, influenza, rhinovirus, and RSV being the most common viral pathogens. In multivariable analysis adjusted for age, sex, and frailty, only high T-reg\% (HR 0.41, 95\% CI 0.20-0.81) and high CMV-reactive CD4+ T cell\% (HR 1.69, 95\% CI 1.03-2.78) were predictive of respiratory viral infection. Notably, CD8 $+\mathrm{T}$ cells were not found to be predictive of respiratory viral infection in the nursing home population.

The fact that high T-regs, which have previously been demonstrated to be increased in the elderly (Johnstone et al. 2014a; Wang et al. 2010), were associated with a reduced risk of infection is unexpected. The authors hypothesized that the increased T-regs may have suppressed immune pathology associated with antiviral immunity.

There has been substantial research examining the role of CMV as a chronic antigenic stimulus responsible for accelerated immunosenescence (Koch et al. 2007; Pawelec et al. 2009). Studies that have examined the effect of CMV infection on response to influenza vaccine response in the elderly has been variable, with some studies showing a negative association and others reporting no impact (den Elzen et al. 2011; Trzonkowski et al. 2003; Derhovanessian et al. 2013). The Canadian study described above focused on the T cell response to CMV and observed that elevated frequencies of CMV-reactive CD4+ T cells, but not CMV-reactive CD8+ $\mathrm{T}$ cells, were associated with an increased risk of respiratory viral infection. One possible interpretation is that the distribution of functional cells (i.e., effector, suppressor, Th1, Th2, etc.) within the CD4+ T cell compartment has a strong influence on host resistance in the elderly.

\section{Conclusions and Future Directions}

This chapter has reviewed the current state of knowledge about immune biomarkers and viral respiratory infections in residents of nursing homes. The chapter provided the reader with an overview of the nursing home environment, highlighting the epidemic nature of respiratory infection in this setting. An overview of biomarkers associated with respiratory viral infections was provided. The nature of this 
environment also makes it difficult to conduct large-scale epidemiological studies. One of the most difficult is enrollment. That is, because the majority of nursing home residents have some degree of cognitive impairment and therefore cannot consent, family members need to be approached. Unfortunately, they often are hard to reach and have little interest in providing consent. Another challenge is that because of confidentiality concerns, the nursing home must first obtain permission from residents' families for the research nurse to approach them. While well intentioned, this also serves as a barrier for recruitment.

Despite these challenges, the nursing home setting is an important one to do research in. The residents of nursing homes are often quite aged, typically frail, and have multiple comorbidities. This offers an important opportunity to evaluate the role of waning immune system in comparison to or when controlled for by age and comorbidity. While it is important to continue to assess immunological difference between younger and older populations, it is important to test the effect of these differences using particular phenotypes. Only by assessing the impact of the immunological differences on clinical outcomes can the relevance of this field of research be made clear. Another challenge of conducting research in this area is identifying the appropriate journals for publication. It is unfortunate that clinical journals fail to grasp the value of including immunologic parameters as risk factors as they do not see this as clinically relevant. Journals that accept more fundamental work generally prefer to accept studies in mice before population-based studies in humans. In both of these cases, this is due to lack of familiarity with the methods, in the first immunological parameters and in the second epidemiologic design and interpretation.

Some of the studies described in this chapter have attempted to link immune biomarkers to clinically important phenotypes, but the majority of studies do not. One reason perhaps is that it is much more difficult to assess residents of nursing homes and to plan studies. The deficits described in the nursing home population highlight the importance of reduced CD8+ naïve T cells, the increase in CD8+ terminally differentiated cells, the increase in $\mathrm{CD} 4+\mathrm{T}$ cells, and the increase in mature NK cells. Evidence of the importance of CD4+ T cells, in particular T-regs and CMV-reactive CD4+ T cells, as a predictor of viral respiratory infection was reviewed. It will be important for investigators to investigate more nursing home cohorts in order to confirm and replicate findings presented by investigators that were reviewed in this chapter. There have been no large-scale epidemiologic studies assessing immune phenotype and influenza vaccine response. This is an important area to investigate given the poor performance of vaccines in this population. Despite new formulations of influenza vaccines, including adjuvant vaccines, increase in hemaglutinin dose, and intradermal vaccines, knowledge about response in large populations is lacking. Investigators conducting clinical trials of influenza vaccine in this population should consider adding blood collection for PBMCs in order to assess biomarkers associated with a robust or poor response. Interaction terms to assess the impact of the presence or absence of the biomarkers should be assessed. 


\section{References}

Bowles SK, Lee W, Simor AE, Vearncombe M, Loeb M, Tamblyn S, Fearon M, Li Y, McGeer A, Oseltamivir Compassionate Use Program Group (2002) Use of oseltamivir during influenza outbreaks in Ontario nursing homes, 1999-2000. J Am Geriatr Soc 50(4):608-616

Centers for Disease Control and Prevention (CDC) (2013) Outbreaks of human metapneumovirus in two skilled nursing facilities -West Virginia and Idaho, 2011-2012. MMWR Morb Mortal Wkly Rep 62(46):909-913

Collerton J, Martin-Ruiz C, Davies K, Hilkens C, Isaacs J, Kolenda C, Parker C, Dunn M, Catt M, Jagger C, von Zglinicki T, Kirkwood T (2012) Frailty and the role of inflammation, immunosenescence and cellular ageing in the very old: cross-sectional findings from the Newcastle 85+ study. Mech Ageing Dev 14:456-466

Cossarizza A, Ortolani C, Paganelli R, Barbieri D, Monti D, Sansoni P, Fagiolo U, Castellani G, Bersani F, Londei M, Franceschi C (1996) CD45 isoforms expression on CD4+ and CD8+ T cells throughout life, from newborns to centenarians: implications for cell memory. Mech Ageing Dev 14:173-195. https://doi.org/10.1016/0047-6374(95)01691-0

Derhovanessian E, Theeten H, Hahnel K, Van Damme P, Cools N et al (2013) Cytomegalovirusassociated accumulation of late-differentiated CD4 T-cells correlates with poor humoral response to influenza vaccination. Vaccine 31:685-690

den Elzen W, Vossen A, Cools H, Westendorp R, Kroes A et al (2011) Cytomegalovirus infection and responsiveness to influenza vaccination in elderly residents of long-term care facilities. Vaccine 29:4869-4874

Eurich DT, Marrie TJ, Johnstone J, Majumdar SR (2008) Mortality reduction with influenza vaccine in patients with pneumonia outside "flu" season: pleiotropic benefits or residual confounding? Am J Respir Crit Care Med 178(5):527-533

Fahey J, Schnelle J, Boscardin J, Thomas J, Gorre ME, Aziz N, Sadeghi H, Nishanian P (2000) Distinct categories of immunologic changes in frail elderly. Mech Ageing Dev 14:1-20

Fulop T, Larbi A, Wikby A, Mocchegiani E, Hirokawa K, Pawelec G (2005) Dysregulation of T-cell function in the elderly : scientific basis and clinical implications. Drugs Aging 22(7):589-603

Gorišek Miksić N, Uršič T, Simonović Z, Lusa L, Lobnik Rojko P, Petrovec M, Strle F (2015) Oseltamivir prophylaxis in controlling influenza outbreak in nursing homes: a comparison between three different approaches. Infection 43(1):73-81

Jackson LA, Jackson ML, Nelson JC, Neuzil KM, Weiss NS (2006a) Evidence of bias in estimates of influenza vaccine effectiveness in seniors. Int J Epidemiol 35(2):337-344

Jackson LA, Nelson JC, Benson P, Neuzil KM, Reid RJ, Psaty BM, Heckbert SR, Larson EB, Weiss NS (2006b) Functional status is a confounder of the association of influenza vaccine and risk of all cause mortality in seniors. Int J Epidemiol 35(2):345-352

Jackson ML, Yu O, Nelson JC, Naleway A, Belongia EA, Baxter R, Narwaney K, Jacobsen SJ, Shay DK, Jackson LA (2013) Further evidence for bias in observational studies of influenza vaccine effectiveness: the 2009 influenza A(H1N1) pandemic. Am J Epidemiol 178 (8):1327-1336

Johnstone J, Millar J, Lelic A et al (2014a) Immunosenescence in the nursing home elderly. BMC Geriatr 14:50. https://doi.org/10.1186/1471-2318-14-50

Johnstone J, Parsons R, Botelho F, Millar J, McNeil S, Fulop T, McElhaney J, Andrew MK, Walter SD, Devereaux PJ, Malekesmaeili M, Brinkman RR, Mahony J, Bramson J, Loeb M (2014b) Immune biomarkers predictive of respiratory viral infection in elderly nursing home residents. PLoS One 9(9):e108481

Koch S, Larbi A, Ozcelik D, Solana R, Gouttefangeas C et al (2007) Cytomegalovirus: a driving force in human T-cell immunosenescence. Ann N Y Acad Sci 1114:23-25

Kupronis BA, Richards CL, Whitney CG, Active Bacterial Core Surveillance Team (2003) Invasive pneumococcal disease in older adults residing in long-term care facilities and in the community. J Am Geriatr Soc 51(11):1520-1525 
Loeb M, McGeer A, McArthur M, Peeling RW, Petric M, Simor AE (2000) Surveillance for outbreaks of respiratory tract infections in nursing homes. CMAJ 162(8):1133-1137

Masse S, Minodier L, Heuze G, Blanchon T, Capai L, Falchi A (2016) Influenza-like illness outbreaks in nursing homes in Corsica, France, 2014-2015: epidemiological and molecular characterization. Springerplus 5(1):1338

McKimm-Breschkin JL (2005) Management of influenza virus infections with neuraminidase inhibitors: detection, incidence, and implications of drug resistance. Treat Respir Med 4 (2):107-116

Meijer A, Overduin P, Hommel D, van Rijnsoever-Greven Y, Haenen A, Veldman-Ariesen MJ (2013) Outbreak of respiratory syncytial virus infections in a nursing home and possible sources of introduction: the Netherlands, winter 2012/2013. J Am Geriatr Soc 61(12):2230-2231

Meydani SN, Leka LS, Fine BC, Dallal GE, Keusch GT, Singh MF, Hamer DH (2004) Vitamin E and respiratory tract infections in elderly nursing home residents: a randomized controlled trial. JAMA 292(7):828-836

Mitchell R, Huynh V, Pak J, Thompson S, Noseworthy AL (2006) Influenza outbreak in an Ontario long-term care home - January 2005. Can Commun Dis Rep 32(21):257-262

Monto AS, Rotthoff J, Teich E, Herlocher ML, Truscon R, Yen HL, Elias S, Ohmit SE (2004) Detection and control of influenza outbreaks in well-vaccinated nursing home populations. Clin Infect Dis 39(4):459-464

Pawelec G, Derhovanessian E, Larbi A, Strindhall J, Wikby A et al (2009) Cytomegalovirus and human immunosenescence. Rev Med Virol 19:47-56

Rivetti D, Jefferson T, Thomas R, Rudin M, Rivetti A, Di Pietrantonj C, Demicheli V (2006) Vaccines for preventing influenza in the elderly. Cochrane Database Syst Rev 3:CD004876

Rockwood K, Abeysundera M, Mitnitski A (2007) How should we grade frailty in nursing home patients? J Am Med Dir Assoc 14:595-603

Saule P, Trauet J, Dutriez V, Lekeux V, Dessaint JP, Labalette M (2006) Accumulation of memory T cells from childhood to old age: central and effector memory cells in CD4(+) versus effector memory and terminally differentiated memory cells in CD8(+) compartment. Mech Ageing Dev 127(3):274-281

Simonsen L, Taylor RJ, Viboud C, Miller MA, Jackson LA (2007) Mortality benefits of influenza vaccination in elderly people: an ongoing controversy. Lancet Infect Dis 7(10):658-666

Strausbaugh LJ, Sukumar SR, Joseph CL (2003) Infectious disease outbreaks in nursing homes: an unappreciated hazard for frail elderly persons. Clin Infect Dis 36(7):870-876

Todd Faulks J, Drinka PJ, Shult P (2000) A serious outbreak of parainfluenza type 3 on a nursing unit. J Am Geriatr Soc 48(10):1216-1218

Trzonkowski P, Mysliwska J, Szmit E, Wieckiewicz J, Lukaszuk K et al (2003) Association between cytomegalovirus infection, enhanced proinflammatory response and low level of anti-hemagglutinins during the anti-influenza vaccination - an impact of immunosenescence. Vaccine 21:3826-3836

Utsumi M, Makimoto K, Quroshi N, Ashida N (2010) Types of infectious outbreaks and their impact in elderly care facilities: a review of the literature. Age Ageing 39(3):299-305

Wack A, Cossarizza A, Heltai S, Barbieri D, D'Addato S, Fransceschi C, Dellabona P, Casorati G (1998) Age-related modifications of the human alphabeta T cell repertoire due to different clonal expansions in the CD4+ and CD8+ subsets. Int Immunol 10(9):1281-1288

Wang L, Xie Y, Zhu L, Chang T, Mao Y et al (2010) An association between immunosenescence and CD4+CD25+ regulatory T-cells: a systematic review. Biomed Environ Sci 23:327-332 